\title{
Graphene Josephson Junction Quantum Circuits for Noise Detection ${ }^{\dagger}$
}

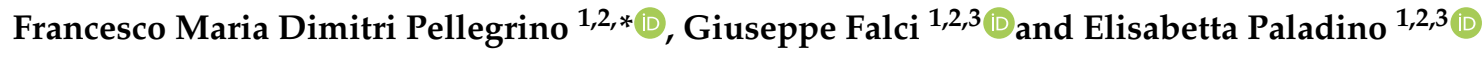 \\ 1 Dipartimento di Fisica e Astronomia "Ettore Majorana", Università di Catania, Via S. Sofia 64, \\ I-95123 Catania, Italy; gfalci@dmfci.unict.it (G.F.); epaladino@dmfci.unict.it (E.P.) \\ 2 INFN, Sez. Catania, I-95123 Catania, Italy \\ 3 CNR-IMM, Via S. Sofia 64, I-95123 Catania, Italy \\ * Correspondence: francesco.pellegrino@ct.infn.it \\ + Presented at the 11th Italian Quantum Information Science conference (IQIS2018), Catania, Italy, \\ 17-20 September 2018.
}

Published: 24 July 2019

\begin{abstract}
Graphene Josephson Junctions (GJJ) in the regime of ballistic transport where current is carried by discrete energy states of Andreev-reflected coherent electron-hole pairs have been recently demonstrated in graphene heterostructures. Due to the non-linear current-phase relation, GJJ can be used as a sensitive probe of underlying microscopic noise sources. Here, we find an analytic expression for the supercurrent-phase relation in the finite low doping regime, and we sketch how a GJJ quantum circuit can play the role of noise quantum sensor.
\end{abstract}

Keywords: Josephson junctions; graphene; superconducting qubits; sensing

\section{Introduction}

The class of devices based on graphene encapsulated in hexagonal boron-nitride (hBN) exhibits pronounced ballistic transport features up to room temperature at a micrometer scale for a wide range of carrier concentration [1-3]. The encapsulation makes graphene almost unaffected by ambient atmosphere and allows the use of boron nitride as an ultrathin top gate dielectric without influencing transport properties of graphene, thanks to ultra-cleanness, large band gap and excellent lattices matching. Combination of these high-quality graphene samples with superconductors via clean interface has led to ballistic transport of Cooper pairs over micron scale lengths [4-6]. Recently, planar graphene Josephson junctions, formed by arranging two superconducting electrodes in close proximity of a graphene layer, have been investigated [7-9]. In a GJJ, a dissipationless supercurrent flows in equilibrium through the normal region via the Andreev reflections at each superconductor-graphene interface. The supercurrent-phase relation deviates significantly from a sinusoidal form [8] with a skewness tunable with the gate voltage [8] and temperature [6]. The Andreev level spectrum, and as a consequence the current-phase relation, depends on the normal phase channel length $(L)$. Here, we focus on the short channel limit, $L \ll \xi$, where $\xi$ is the coherence length in the superconductor. This regime has been recently experimentally achieved in [9] and accurately described within the Dirac-Bogoliubov-de Gennes approach [10]. Because of the mentioned characteristics, GJJ are also an attractive alternative for the realization of quantum circuits, possibly microwave driven, for quantum computing purposes [11]. Recently, a graphene-based transmon qubit insensitive to parallel large magnetic field has been realized [12].

In this manuscript, we derive low doping corrections to the current-phase relation at zero temperature in short ballistic GJJ, based on Dirac-Bogoliubov-de Gennes approach [10]. We demonstrate that embedding the GJJ in a quantum circuit with DC current bias, a GJJ-phase 
qubit may be envisaged allowing for sensing [13,14] of pure dephasing critical current noise [15] induced by carrier density fluctuations.

\section{Current-Phase Relation for Finite Doping at $T=0$}

We consider a GJJ formed by single layer of graphene in the $x-y$ plane, covered by superconducting electrodes in the regions $x<-L / 2$ and $x>L / 2$. Along the $\hat{y}$ direction the sample has a width $W$ and it is uniform. The graphene sectors covered by superconducting stripes behave as superconducting metals thanks to the proximity effect. We focus on wide and short junctions, namely the length $L$ of the junction is much smaller than the superconducting coherence length $\xi$ and sample width $W$. Under this condition, the GJJ can be modelled within the Dirac-Bogoliubov-de Gennes approach [10] resulting in a supercurrent at zero temperature given by

$$
I\left(k_{\mathrm{F}}, \phi\right) \equiv-\frac{4 e}{\hbar} \frac{W}{\pi} \int_{0}^{\infty} d q \frac{\partial \varepsilon\left(k_{\mathrm{F}}, q, \phi\right)}{\partial \phi},
$$

where $\phi$ is the phase difference between the superconductors on the two sides of the graphene stripe. The Andreev bound state energies, assuming that the lateral superconductive sides have identical gap $\Delta_{0}$, read

$$
\varepsilon\left(k_{\mathrm{F}}, q, \phi\right)=\Delta_{0} \sqrt{1-\tau\left(k_{\mathrm{F}}, q\right) \sin ^{2}(\phi / 2)},
$$

where the normal state transmission probability reads

$$
\tau\left(k_{\mathrm{F}}, q\right)=\frac{k_{\mathrm{F}}^{2}-q^{2}}{k_{\mathrm{F}}^{2}-q^{2} \cos ^{2}\left(\sqrt{k_{\mathrm{F}}^{2}-q^{2}} L\right)},
$$

and $k_{\mathrm{F}}=\sqrt{\pi\left|n_{0}\right|}$ is the Fermi wavevector $\left(n_{0}\right.$ is the carrier density, it is positive for electron doping and negative for hole doping).

Since we are interested in low doping regime, $\left|n_{0}\right| \ll 1 / L^{2} \approx 10^{10} \mathrm{~cm}^{-2}$, we start from the pristine graphene case, i.e., without free carriers $\left(n_{0}=0\right)$. In this case supercurrent Equation (1) takes the following analytical form [10]

$$
I_{0}(\phi) \equiv I(0, \phi)=\frac{e \Delta_{0} W}{\hbar L} \frac{2}{\pi} \operatorname{atanh}[\sin (\phi / 2)] \cos (\phi / 2),
$$

whose maximum value, attained at $\phi^{*} \approx 1.97$, represents the Josephson critical current

$$
I_{0 \mathrm{c}}=\frac{e \Delta_{0} W}{\hbar L} \frac{1.33}{\pi}
$$

By expanding the argument of the integral in Equation (1) with respect to the Fermi wave vector around the neutrality point $\left(k_{\mathrm{F}}=0\right)$, we find

$$
I\left(k_{\mathrm{F}}, \phi\right) \approx I_{0}(\phi)+\mathcal{J}(\phi)\left(k_{\mathrm{F}} L\right)^{2},
$$

where

$$
\mathcal{J}(\phi)=\frac{e \Delta_{0}}{\hbar} \frac{W}{\pi L} \int_{0}^{\infty} d x \frac{\sin (\phi)[x-\tanh (x)] \tanh (x)[2 \cosh (2 x)+\cos (\phi)+1]}{\sqrt{2} x^{2} \cosh (x)[\cosh (2 x)+\cos (\phi)]^{3 / 2}} .
$$

In Figure 1a, we report $\mathcal{J}(\phi)$ as a function of the phase $\phi$ (black solid line) and $\mathcal{J}_{a_{0}}(\phi)=a_{0} I_{0}(\phi)$ with $a_{0}=0.112$ (red dashed line). Remarkably, the phase-dependence of the finite doping correction factor strictly follows the undoped current-phase relation, $I_{0}(\phi)$, allowing the approximation of supercurrent for low doping as

$$
I\left(k_{\mathrm{F}}, \phi\right) \approx\left[1+a_{0}\left(k_{\mathrm{F}} L\right)^{2}\right] I_{0}(\phi) .
$$


In turn, the critical current depends on the doping concentration and it is approximately given by

$$
I_{c} \approx\left[1+a_{0}\left(k_{\mathrm{F}} L\right)^{2}\right] I_{0 \mathrm{c}}=\frac{1.33}{\pi} \frac{e \Delta_{0} W}{\hbar L}\left[1+a_{0}\left(k_{\mathrm{F}} L\right)^{2}\right],
$$

where $I_{0 \mathrm{c}}$ is given by Equation (5). In Figure $1 \mathrm{~b}$ we plot the critical current as a function of the Fermi level $\mu_{0}=\hbar v \sqrt{\pi n_{0}}$ (black solid line), where $v \approx 10^{6} \mathrm{~m} / \mathrm{s}$, obtained by maximing the expansion in Equation (6) and compare with the approximate critical current given by Equation (9) (red dashed). We conclude that the analytical expression in Equation (9) is an excellent approximation of the critical current for low doping.

(a)

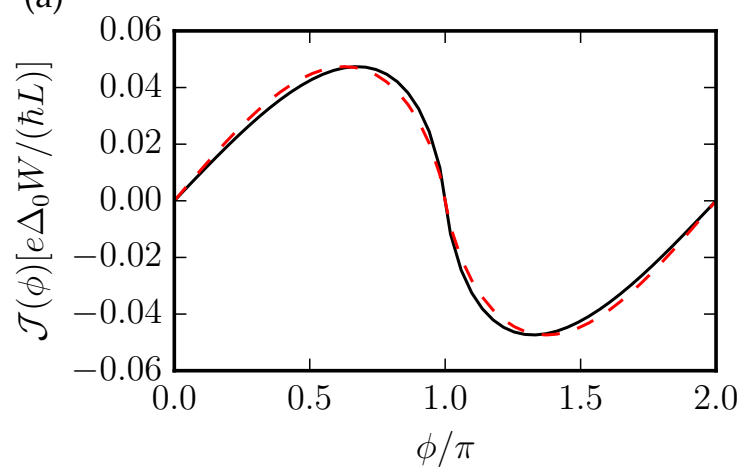

(b)

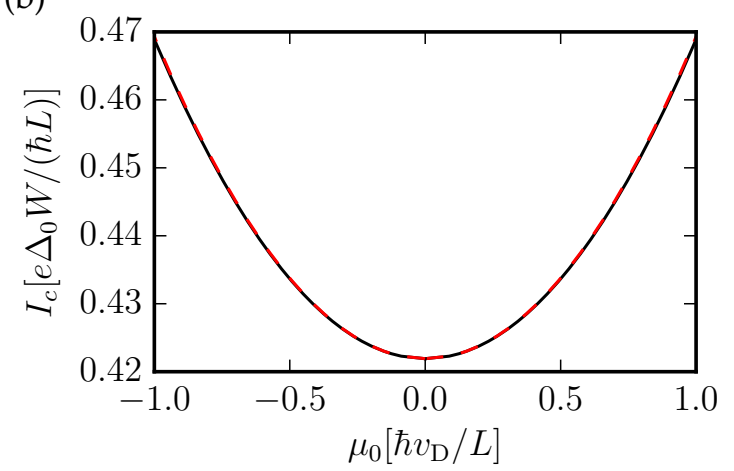

Figure 1. Panel (a): $\mathcal{J}(\phi)$ defined in Equation (7) (solid black line) and $\mathcal{J}_{a_{0}}(\phi)=a_{0} I_{0}(\phi)$ with $a_{0}=0.112$ (dashed red line) in units of $e \Delta_{0} W /(\hbar L)$ as a function of the phase $\phi$. Panel (b): Critical current, in units of $e \Delta_{0} W /(\hbar L)$, at low doping as a function of the Fermi level at zero temperature. The solid black line refers to the critical current obtained by maximing expression in Equation (6), the dashed red line is the approximation of the critical current given by Equation (9).

\section{GJJ Phase Qubit for Noise Sensing}

A GJJ can be embedded in a quantum circuit with a DC current bias, analogously to conventional Josephson junctions [16,17]. The circuit can be described by the RCSJ model [18], whose non dissipative part consists of a particle of mass corresponding to the junction's capacitance, $C$, in a tilted washboard potential expressed in terms of the bias current $I_{\mathrm{b}}$ and the supercurrent via $F(\phi) \equiv \frac{1}{I_{\mathrm{C}}} \int d \phi I\left(k_{\mathrm{F}}, \phi\right)$

$$
\mathcal{H}=\frac{Q^{2}}{2 C}+\frac{\hbar}{2 e} I_{\mathrm{C}} F(\phi)-\frac{\hbar}{2 e} I_{\mathrm{b}} \phi .
$$

The junction charging energy, $Q^{2} / 2 C$, where $Q$ is the charge accumulated on the two sides of the junction, represents a kinetic energy contribution. When the bias current approaches the critical current $I_{\mathrm{b}} \leq I_{\mathrm{c}}$, the tilted washboard potential has a minimum close to $\phi^{*}$ (the phase difference value where the maximum of the supercurrent is located). Around the minimum the potential can be approximated by the cubic form $U(\phi)=\frac{k}{2}\left(\phi-\phi_{0}\right)^{2}+\lambda\left(\phi-\phi_{0}\right)^{3}$, where $\phi_{0}=\phi^{*}+\sqrt{2\left(1-I_{\mathrm{b}} / I_{\mathrm{c}}\right) / \alpha_{\mathrm{s}}}$, $k=\frac{\hbar}{2 e} I_{\mathrm{c}} \sqrt{2\left(1-I_{\mathrm{b}} / I_{\mathrm{c}}\right) \alpha_{\mathrm{s}}}, \lambda=-\frac{\hbar}{12 e} I_{\mathrm{c}} \alpha_{\mathrm{s}}$ and $\alpha_{\mathrm{s}}=-\left.\frac{d^{3} F(\phi)}{d \phi^{3}}\right|_{\phi=\phi^{*}}$. Heisenberg quantization of the conjugate variables, charge $Q$ and phase $\phi$, implies discretized levels of the anharmonic potential (underdamped regime of the RCSJ model). Treating the anharmonicity term within a perturbative approach, we estimate the eigenenergies as

$$
E_{n} \approx \hbar \omega_{\mathrm{P}}\left(n+\frac{1}{2}\right)-\frac{e^{2}}{C} \frac{5 \alpha_{\mathrm{s}}}{24\left(1-I_{\mathrm{b}} / I_{\mathrm{c}}\right)}\left(n^{2}+n+\frac{11}{30}\right)
$$

where $\omega_{\mathrm{p}}=\sqrt{\frac{2 e I_{\mathrm{C}}}{\hbar C}}\left[2\left(1-I_{\mathrm{b}} / I_{\mathrm{c}}\right) \alpha_{\mathrm{s}}\right]^{1 / 4}$ and $n \in \mathbb{N}$. By referring to the supercurrent-phase relation in Equation (8) one has $\alpha_{\mathrm{s}} \approx 0.82$, at odds with the sinusoidal supercurrent-phase relation where 
$\alpha_{\mathrm{S}}$ is exactly one [16]. The anharmonicity is easily estimated as $\frac{E_{n+1}-E_{n}-\hbar \omega_{\mathrm{P}}}{E_{1}-E_{0}-\hbar \omega_{\mathrm{P}}}=n+1$, the linear increase with $n$ of the splitting between neighboring levels being due to anharmonic potential term. This result suggests that the two lowest energy levels of the anharmonic potential may be employed as a GJJ-phase qubit with a splitting dependent on the GJJ critical current given by Equation (9). Additional AC currents via the same $I_{b}$ port would allow qubit state manipulation [17]. Fluctuations of the critical current, induced for instance by carrier density variations, directly enter the level splitting of the GJJ phase qubit inducing a pure dephasing effect. Pure dephasing due to low frequency fluctuations represents a severe limitation to the qubit coherent evolution. Whenever present, pure dephasing effects are more detrimental that possible dissipative transitions requiring incoherent energy exchanges between system and environment [19]. Low frequency noise in the Josephson critical current in conventional superconducting qubits has been the subject of several investigations [15]. On a complementary perspective, noise sensitivity of superconducting qubits can be exploited for noise spectroscopy [14,15,20-24]. A number of experiments demonstrated the capabilities of suitably designed multipulse control protocols for filtering environmental noise, see for instance [25-28]. Based on these results, here we suggest the use of a GJJ-phase qubit for sensing of critical current noise in the GJJ. To this end, we restrict the analysis to the bidimensional lowest energy subspace where

$$
H=\left[\frac{\hbar \omega_{10}}{2}+b(t)\right] \sigma_{z}+\frac{g(t)}{2} e^{i \omega_{10} t} \sigma_{+}+\frac{g(t)}{2} e^{-i \omega_{10} t} \sigma_{-},
$$

here $\omega_{10}=\left(E_{1}-E_{0}\right) / \hbar$ is the energy splitting with pure dephasing effects due to critical current fluctuations originated by $b(t)=\frac{\hbar}{2} \frac{\partial \omega_{10}}{\partial I_{\mathrm{C}}} \delta I_{\mathrm{C}}(t)$. The control via DC and AC pulses is included in the term $g(t)=\frac{\hbar}{e} \sqrt{\frac{\hbar \omega_{10}}{2 k}} I_{\chi}(t)$ realized via the AC current bias. Assuming Gaussian critical current fluctuations, the qubit coherences decay as $[15,29]$

$$
\left\langle\sigma_{+}(t)\right\rangle=\left\langle\sigma_{+}(0)\right\rangle e^{-i \omega_{10} t-\mathcal{J}(t)}
$$

where the decay factor depends on the critical current power spectrum $\mathcal{S}_{\delta I_{\mathrm{c}}}(\omega)=\int_{0}^{\infty} \frac{d t}{\pi}\left\langle\delta I_{\mathrm{C}}(t) \delta I_{\mathrm{c}}(0)\right\rangle \cos (\omega t)$ as

$$
\mathcal{J}(t)=\frac{1}{4}\left(\frac{\partial \omega_{10}}{\partial I_{\mathrm{C}}}\right)^{2} \int_{-\infty}^{+\infty} d \omega \mathcal{S}_{\delta I_{\mathrm{C}}}(\omega) \mathcal{F}_{t}(\omega)
$$

The function $\mathcal{F}_{t}(\omega)$

$$
\mathcal{F}_{t}(\omega)=\mid \int_{0}^{t} d \tau e^{i \omega \tau} e^{-\left.\frac{i}{e} \sqrt{\frac{\hbar \omega_{10}}{2 k}} \int_{0}^{\tau} d \tau^{\prime} I_{X}\left(\tau^{\prime}\right)\right|^{2},}
$$

plays the role of a filter [30] selecting specific frequency ranges of the critical current power spectrum $\mathcal{S}_{\delta I_{\mathrm{c}}}(\omega)$ by a suitable choice of the external control. The form (14) of the dephasing factor is well known in the literature $[14,30]$. With the analytic form for the GJJ critical current we derived in (9) it points out the possibility to employ quantum circuits based on GJJ for noise sensing in graphene in the ballistic transport regime.

\section{Conclusions}

In this manuscript, within a Dirac-Bogoliubov-de Gennes approach [10] we have obtained an analytical, but non-sinusoidal, expression for the supercurrent-phase relation in a wide short ballistic GJJ for small, finite doping. The approximate form given in Equation (9) explicitly relates the critical current to the doping concentration. Finally, we have sketched how a GJJ phase qubit might be employed for noise sensing of ballistic transport noise in graphene via the qubit's evolution assisted by a suitable external control. 


\section{References}

1. Wang, L.; Meric, I.; Huang, P.Y.; Gao, Q.; Gao, Y.; Tran, H.; Taniguchi, T.; Watanabe, K.; Campos, L.M.; Muller, D.A.; Guo, J.; Kim, P.; Hone, J.; Shepard, K.L.; Dean, C.R. One-Dimensional Electrical Contact to a Two-Dimensional Material. Science 2013, 342, 614, doi:10.1126/science.1244358.

2. Dean, C.R.; Young, A.F.; Meric, I.; Lee, C.; Wang, L.; Sorgenfrei, S.; Watanabe, K.; Taniguchi, T.; Kim, P.; Shepard, K.L.; Hone, J. Boron nitride substrates for high-quality graphene electronics. Nat. Nanotech. 2010, 5 , 722-726, doi:10.1038/nnano.2010.172.

3. Mayorov, A.S.; Gorbachev, R.V.; Morozov, S.V.; Britnell, L.; Jalil, R.; Ponomarenko, L.A.; Blake, P.; Novoselov, K.S.; Watanabe, K.; Taniguchi, T.; Geim, A.K. Micrometer-Scale Ballistic Transport in Encapsulated Graphene at Room Temperature. Nano Lett. 2011, 11, 2396-2399, doi:10.1021/nl200758b.

4. Shalom, M.B.; Zhu, M.J.; Fal'ko, V.I.; Mishchenko, A.; Kretinin, A.V.; Novoselov, K.S.; Woods, C.R.; Watanabe, K.; Taniguchi, T.; Geim, A.K.; Prance, J.R. Quantum oscillations of the critical current and high-field superconducting proximity in ballistic graphene. Nature Phys. 2015, 12, 318-322, doi:10.1038/nphys.3592.2016.

5. Calado, V.E.; Goswami, S.; Nanda, G.; Diez, M.; Akhmerov, A.R.; Watanabe, K.; Taniguchi, T.; Klapwijk, T.M.; Vandersypen, L.M.K. Ballistic Josephson junctions in edge-contacted graphene. Nat. Nano 2015, 10, 761, doi:10.1038/nnano.2015.156.

6. English, C.D.; Hamilton, D.R.; Chialvo, C.; Moraru, I.C.; Mason, N.; van Harlingen, D.J. Observation of nonsinusoidal current-phase relation in graphene Josephson junctions. Phys. Rev. B 2016, 94, 115435, doi:10.1103/PhysRevB.94.115435.

7. Borzenets, I.V.; Amet, F.; Ke, C.T.; Draelos, A.W.; Wei, M.T.; Seredinski, A.; Watanabe, K.; Taniguchi, T.; Bomze, Y.; Yamamoto, M.; et al. Ballistic Graphene Josephson Junctions from the Short to the Long Junction Regimes. Phys. Rev. Lett. 2016, 117, 237002, doi:10.1103/PhysRevLett.117.237002.

8. Nanda, G.; Aguilera-Servin, J.L.; Rakyta, P.; Kormanyos, A.; Kleiner, R.; Koelle, D.; Watanabe, K.; Taniguchi, T.; Vandersypen, L.M.K.; Goswami, S. Current-Phase Relation of Ballistic Graphene Josephson Junctions. Nano Lett. 2017, 17, 3396-3401, doi:10.1021/acs.nanolett.7b00097.

9. Park, J.; Lee, J.H.; Lee, G.-H.; Takane, Y.; Imura, K.-I.; Taniguchi, T.; Watanabe, K.; Lee, H.-J. Short Ballistic Josephson Coupling in Planar Graphene Junctions with Inhomogeneous Carrier Doping. Phys. Rev. Lett. 2018, 120, 077701, doi:10.1103/PhysRevLett.120.077701.

10. Titov, M.; Beenakker, C.W.J. Josephson effect in ballistic graphene. Phys. Rev. B 2006 74, 041401, doi:10.1103/PhysRevB.74.041401.

11. Schmidt, F.E.; Jenkins, M.D.; Watanabe, K.; Taniguchi, T.; Steele, G.A. A ballistic graphene superconducting microwave circuit. Nat. Commu. 2018, 9, 4069, doi:10.1038/s41467-018-06595-2.

12. Kroll, J.G.; Schmidt, F.E.; Jenkins, M.D.; Watanabe, K.; Taniguchi, T.; Steele, G.A. Magnetic field compatible circuit quantum electrodynamics with graphene Josephson junctions. Nat. Commu. 2018, 9, 4615, doi:10.1038/s41467-018-07124-x.

13. Zwick, A.; Alvarez, G.A.; Kurizki, G.; Maximizing Information on the Environment by Dynamically Controlled Qubit Probes. Phys. Rev. Appl. 2016, 5, 014007, doi:10.1103/PhysRevApplied.5.014007.

14. Degen, C.L.; Reinhard, F.; Cappellaro, P. Quantum sensing. Rev. Mod. Phys. 2017, 89, 035002, doi:10.1103/RevModPhys.89.035002.

15. Paladino, E.; Galperin, Y.M.; Falci, G.; Altshuler, B.L. $1 / f$ noise: Implications for solid-state quantum information. Rev. Mod. Phys. 2014, 86, 361, doi:10.1103/RevModPhys.86.361.

16. Martinis, J.M.; Nam, S.; Aumentado, J.; Urbina, C. Rabi Oscillations in a Large Josephson-Junction Qubit. Phys. Rev. Lett. 2002, 89, 117901, doi:10.1103/PhysRevLett.89.117901.

17. Martinis, J.M.; Nam, S.; Aumentado, J.; Lang, K.M.; Urbina, C. Decoherence of a superconducting qubit due to bias noise. Phys. Rev.B 2003, 67, 094510, doi:10.1103/PhysRevB.67.094510.

18. Devoret, M.H.; Martinis, J.M.; Clarke, J. Measurements of Macroscopic Quantum Tunneling out of the Zero-Voltage State of a Current-Biased Josephson Junction. Phys. Rev. Lett. 1985, 55, 1908, doi:10.1103/PhysRevLett.55.1908.

19. Paladino, E.; D'arrigo, A.; Mastellone, A.; Falci, G. Decoherence times of universal two-qubit gates in the presence of broad-band noise. New J. Phys. 2011, 13, 09037, doi:10.1088/1367-2630/13/9/093037. 
20. Faoro, L.; Viola, L. Dynamical suppression of 1 /f noise processes in qubit systems. Phys. Rev. Lett. 2004, 92, 117905, doi:10.1103/PhysRevLett.92.117905.

21. Falci, G.; D'Arrigo, A.; Mastellone, A.; Paladino, E. Dynamical suppression of telegraph and $1 / \mathrm{f}$ noise due to quantum bistable fluctuators. Phys. Rev. A 2004, 70, 040101, doi:10.1103/PhysRevA.70.040101.

22. Cywinski, L.; Lutchyn, R.M.; Nave, C.P.; Sarma, S.D. How to enhance dephasing time in superconducting qubits. Phys. Rev. B 2008, 77, 174509, doi:10.1103/PhysRevB.77.174509.

23. D'Arrigo, A.; Falci, G.; Paladino, E. Quantum Zeno and anti-Zeno effect on a two-qubit gate by dynamical decoupling. EPJ-ST 2019, 227, 2189, doi:10.1140/epjst/e2018-800081-0.

24. D'Arrigo, A.; Falci, G.; Paladino, E. High-fidelity two-qubit gates via dynamical decoupling of local $1 / f$ noise at the optimal point. Phys. Rev. A 2016, 94, 022303, doi:10.1103/PhysRevA.94.022303.

25. Biercuk, M.J.; Doherty, A.C.; Uys, H. Dynamical decoupling sequence construction as a filter-design problem. J. Phys. B: At. Mol. Opt. Phys. 2011, 44, 154002, doi:10.1088/0953-4075/44/15/154002.

26. Bylander, J.; Gustavsson, S.; Yan, F.; Yoshihara, F.; Harrabi, K.; Fitch, G.; Cory, D.G.; Nakamura, Y.; Tsai, J.-S.; Oliver, W.D. Noise spectroscopy through dynamical decoupling with a superconducting flux qubit. Nat. Phys. 2011, 7, 565, doi:10.1038/nphys1994.

27. Yuge, T.; Sasaki, S.; Hirayama, Y. Measurement of the noise spectrum using a multiple-pulse sequence. Phys. Rev. Lett. 2011, 107, 170504, doi:10.1103/PhysRevLett.107.170504.

28. Yan, F.; Bylander, F.; Gustavsson, S.; Yoshihara, F.; Harrabi, K.; Cory, D.G.; Orlando, T.P.; Nakamura, Y.; Tsai, J.-S.; Oliver, W.D. Spectroscopy of low-frequency noise and its temperature dependence in a superconducting qubit. Phys. Rev. B 2012, 85, 174521, doi:10.1103/PhysRevB.85.174521.

29. Palma, G.; Suominen, K.; Ekert, A. Quantum computers and dissipation. Proc. R. Soc. A 1996 452, 567, doi:10.1098/rspa.1996.0029.

30. Kofman, A.G.; Kurizki, G. Universal Dynamical Control of Quantum Mechanical Decay: Modulation of the Coupling to the Continuum. Phys. Rev. Lett. 2001, 87, 270405, doi:10.1103/PhysRevLett.87.270405.

(C) 2018 by the authors. Licensee MDPI, Basel, Switzerland. This article is an open access article distributed under the terms and conditions of the Creative Commons Attribution (CC BY) license (http:/ / creativecommons.org/licenses/by/4.0/). 